\title{
A GROUP ANALYTIC NETWORK PROCESS (ANP) FOR INCOMPLETE INFORMATION
}

\author{
Kouichi Taji, Yousuke Sagayama ${ }^{\dagger}$ and Hiroyuki Tamura \\ Department of Systems and Human Science, Graduate School of Engineering Science, \\ Osaka University, 1-3 Machikaneyama, Toyonaka, Osaka 560-8531, Japan \\ \{taji,tamura\}@sys.es.osaka-u.ac.jp
}

Keywords: group decision-making, ANP, supermatrix method, incomplete information

Summary: In this paper, we propose an ANP framework for group decision-making problem with incomplete information. The proposed method consists of three parts, namely, aggregation of weights of alternatives, aggregation of weights of criteria and evaluation of global weights. In aggregating weights of alternatives, we calculate group weights from individual weights, which have been calculated by some AHP, by using a quadratic programming problem. In aggregating the weights of criteria, we obtain the weights for each alternative by using the geometrical average method for pairwise comparison matrices of decision-makers only who evaluate the alternative. Then the weights of criteria may be different in each alternative. Hence, we use the supermatrix method of ANP for evaluating global weights. A small numerical example shows that our proposed framework enables us to obtain the global weights of a group decision-making problem with incomplete information.

\section{Introduction}

The AHP (Analytic Hierarchy Process), proposed by Saaty (1980), is one of decision making models which consists of three parts, namely, making hierarchy structure of the problem, evaluating local weights by pairwise comparison and evaluating the global weights by additional sum. An AHP has been widely used because it can deal with unquantifiable objects and its implementation is very easy. In some practical decision problem, it seems to be the case where local weights of criteria are different for every alternative. An AHP has a difficulty to treat such a case because an AHP uses the same local weights of criteria for each alternative.

To overcome this difficulty, Saaty (1996) proposed an ANP (Analytic Network Process). An ANP permits to use different weighs of criteria for alternatives. But in this case, global weights cannot be evaluated by additive sum as in AHP, and hence, Saaty introduced a so-called supermatrix and he proposed that global weights are the eigenvector corresponding to the maximum eigenvalue of a supermatrix. When all local weight vectors of criteria are same, an ANP is known to reduce to an AHP. Therefore, an ANP includes an AHP as a special case and has ability to treat more various decision problems than AHP. Decision problems seen in companies and politics tend to be large and complicated, and more than one decision-makers often must participate to decide. Both an AHP and an ANP are the method supporting for personal decision-making, and it has been required to extend these two methods to supporting group decision-making problems.

Various modifications of AHP, such as the geometrical average method, the interval AHP (Yamada et al., 1997) and the stress method (Nakanishi and Kinoshita, 1999), have been proposed for group decisionmaking problem. In these methods, each decision-maker first makes his/her pairwise comparison matrices, next these matrices are aggregated to group pairwise comparison matrices, and then global

\footnotetext{
${ }^{\dagger}$ Presently with Nissha Printing Co., Ltd., 3 Mibu Hanai-cho, Nakagyo-ku, Kyoto 604-8551, Japan. y-sagayama@is.nissha.co.jp
} 
weights of group are evaluated by an eigenvector method. However, in the group decision-making, there may often be the case that all decision makers do not necessarily evaluate all alternatives. This case is called incomplete information (Harker 1987) and pairwise comparison matrices have some blanks. Since a geometrical average method and the interval AHP assume complete pairwise comparison, they cannot obtain global weights for incomplete information cases. To overcome this difficulty, Yamaki and Sekitani have proposed the large scale AHP (Yamaki and Sekitani, 1999). In the large scale AHP, weights are obtained as a solution to a certain quadratic programming problem, which is equivalent to the eigenvalue method in some sense.

In this paper, we propose an ANP framework for group decision-making problem with incomplete information. So far as the authors know, an ANP has not been extended to group decision-making. The method uses local weights of alternatives, which each decision-maker has calculated by a certain AHP, and group weights of criteria for each alternative are calculated from pairwise comparison matrices of decision makers only who evaluate the alternative. Then since the weights of criteria may be different in each alternative, we use the supermatrix method of ANP for evaluating global weights.

This paper is organized as follows. In section 2, we explain our proposed method in detail. The method is applied to a small numerical example with incomplete information in Section 3. Finally in section 4, we conclude the method and summarize subjects of future research.

\section{Group ANP}

The proposed method consists of three parts, namely, aggregation of weights of alternatives, aggregation of weights of criteria and evaluation of global weights. In this section, we explain these three parts in detail. Throughout this paper, we consider the case that $n$ decision makers, $D M_{i}(i=1, \ldots, n)$, evaluate $m$ alternatives, $A_{j}(j=1, \ldots, m)$, under $l$ criteria, $C_{k}(k=1, \ldots, l)$. We assume that each decision maker need not necessarily evaluate all alternatives, but when a decision-maker evaluates an alternative, its evaluation is performed under all criteria.

\subsection{Aggregation of weights of alternatives}

In the case of incomplete information, it is not easy to aggregate pairwise comparison matrices of decision-makers to one matrix because each comparison matrices may have blanks. Hence, each decision-maker is to evaluate under every criteria local weights of alternatives only which he/she can evaluate. We consider aggregating these local weights to local weights of the group.

Let $a_{i j}^{k}$ denote a local weight of a decision-maker $D M_{i}$ evaluating an alternative $A_{j}$ under a criterion $C_{k}$. We note that $a_{i j}^{k}>0$ holds whenever $D M_{i}$ evaluates $A_{j}$ and otherwise $a_{i j}^{k}=0$. We also remark that the normalization procedure $\sum_{j=1}^{m} a_{i j}^{k}=1$ is not necessarily assumed because the proposed aggregation method is shown to be independent of normalization methods.

We introduce the following bipartite graph. Two node sets consist of the set $I=\{1, \ldots, n\}$ and $J=\{1, \ldots, m\}$ corresponding to decision-makers and alternatives, respectively, and the arc $(i, j)$, $i \in I$ and $j \in J$, exists if and only if $D M_{i}$ evaluates $A_{j}$, that is, the arc $(i, j)$ exists if and only if $a_{i j}^{k}>0$. We call this graph evaluation graph.

(Example) We consider the case of three decision-makers and three alternatives. A decision-maker $D M_{1}$ evaluates alternatives $A_{1}$ and $A_{2}, D M_{2}$ evaluates all alternatives and that $D M_{3}$ evaluates $A_{2}$ and 
$A_{3}$. Then local weights $a_{1 j}^{k}(j=1,2), a_{2 j}^{k}(j=1,2,3)$ and $a_{2 j}^{k}(j=2,3)$ are positive and the other local weights are zero. An evaluation graph of this example is shown in Figure 1.

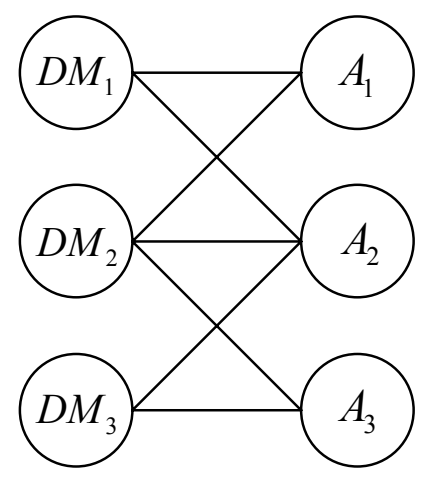

Figure 1. Evaluation graph

In the definition above, an evaluation graph may not be connected in the sense of graph theory. For the unconnected case, we recommend that local weights of alternatives are first obtained separately on each connected components and then, by a certain methods agreed by decision-makers, whole results are calculated from these weights. Therefore, in the rest of this paper, we consider only the case that an evaluation graph is connected.

In group decision-making problems, all decision-makers do not necessarily evaluate all alternatives. Furthermore, if each decision-maker uses different normalization procedure of his/herself, the absolute values of weights are variable according to the number of alternatives evaluated by a decision-maker and a normalization procedure. It has been considered that an AHP is the method evaluating alternatives in a ratio scale rather than interval scale (Harker and Vargas, 1987). According to this, it may be desirable that weights of a group are selected such that the ratio of the weights for alternatives is as close as possible to that of individual weights. That is, if local weights of two decision-makers satisfy $a_{i j}^{k} / a_{i j^{\prime}}^{k}=a_{i^{\prime} j}^{k} / a_{i^{\prime} j^{\prime}}^{k}$, then it is desirable that a group weights $x^{k}$ also satisfies $x_{j}^{k} / x_{j^{\prime}}^{k}=a_{i j}^{k} / a_{i j^{\prime}}^{k}=a_{i^{\prime} j}^{k} / a_{i^{\prime} j^{\prime}}^{k}$.

Based on the above observations, we propose an aggregation method in which a group local weights vector of alternatives $x^{k}=\left(x_{1}^{k}, \ldots, x_{n}^{k}\right)^{T}$ under a criterion $k$ is obtained as the solution $x^{*}$ to the following quadratic programming problem:

$$
\begin{array}{ll}
\min _{w, x} & \frac{1}{2} \sum_{i=1}^{n} \sum_{j=1}^{m}\left(a_{i j}^{k} w_{i}-\operatorname{sgn}\left(a_{i j}^{k}\right) x_{j}\right)^{2} \\
\text { s.t. } & \sum_{j=1}^{m} x_{j}=1, \quad w_{i} \geq 0(i=1, \ldots, n), x_{j} \geq 0(j=1, \ldots, m),
\end{array}
$$

where $\operatorname{sgn}(\cdot)$ is a function defined as

$$
\operatorname{sgn}(t)=\left\{\begin{array}{cc}
1 & t>0 \\
0 & t=0 \\
-1 & t<0
\end{array}\right.
$$


The objective function of (1) is the square sum of differences between a group weight $x_{j}$ and an individual weight $a_{i j}^{k}$ multiplied by a modification factor $w_{i}$, and hence, it can be considered as a dissatisfaction value.

When an evaluation graph is connected, the following theorem holds for the optimization problem (1).

Theorem 1. When an evaluation graph is connected, the optimization problem (1) has a unique pair of solutions $\left(w^{*}, x^{*}\right)$. Furthermore, $w^{*}>0$ and $x^{*}>0$.

Remark. This theorem guarantees that, under a connected assumption, group weights are determined uniquely. Moreover, since modification factors $w_{i}$ are all positive, estimation of all decision-makers is necessarily reflected on aggregated group weights in some extent. Also, since group weights $x_{j}$ are all positive, there is no alternative ignored by a group.

Proof of Theorem 1. To simplify the notation, we ignore the super script ${ }^{k}$. We first show $w^{*}>0$ and $x^{*}>0$. It is easy to see that the problem (1) is equivalent to the optimization problem

$$
\begin{array}{ll}
\min _{w, x} & \frac{1}{2} \sum_{i=1}^{n} \sum_{j=1}^{m}\left(a_{i j} w_{i}-\operatorname{sgn}\left(a_{i j}\right) x_{j}\right)^{2} \\
\text { s.t. } & \sum_{j=1}^{m} x_{j} \geq 1, \quad w_{i} \geq 0(i=1, \ldots, n), x_{j} \geq 0(j=1, \ldots, m),
\end{array}
$$

where the constraint $\sum_{j=1}^{m} x_{j}=1$ is relaxed. Hence, there exist Lagrange multipliers $\lambda, \mu$ and $\pi$ such that solution to (1) satisfies the Karush-Kuhn-Tucker condition for (2), that is,

$$
\begin{aligned}
& \lambda_{i}=\sum_{j=1}^{m}\left(a_{i j}^{2} w_{i}-a_{i j} x_{j}\right) \geq 0,(i=1, \ldots, n), \\
& \mu_{j}=-\sum_{i=1}^{n}\left(a_{i j} w_{i}-\operatorname{sgn}\left(a_{i j}\right) x_{j}\right)-\pi \geq 0,(j=1, \ldots, m), \\
& \pi\left(\sum_{j=1}^{m} x_{j}-1\right)=0, \quad \lambda^{T} w=0, \quad \mu^{T} x=0, \\
& \sum_{j=1}^{m} x_{j} \geq 1, \quad w \geq 0, \quad x \geq 0, \quad \pi \geq 0 .
\end{aligned}
$$

Suppose that $w_{s}^{*}=0$. Then we have from (3a) that

$$
\sum_{j=1}^{m} a_{s j} x_{j}^{*} \leq 0 .
$$

Hence, we must have $a_{s j} x_{j}^{*}=0$ for all $j \in J$ because $a_{s j} \geq 0$ and $x_{j}^{*} \geq 0$. If there exists an arc $(s, j)$ in an evaluation graph, $a_{s j}>0$ holds, and hence, we have $x_{j}^{*}=0$. Thus $x_{j}^{*}=0$ for all nodes $j \in J$ adjacent to the node $s$. Suppose also that $x_{t}^{*}=0$. Since $\pi \geq 0$, it follows from (3b) that

$$
\sum_{i=1}^{n} a_{i t} w_{i}^{*} \leq 0 \text {. }
$$


It follows from the same argument above that $w_{i}^{*}=0$ for all nodes $i \in I$ adjacent to the node $t$.

Therefore, since an evaluation graph is connected, if one of $w_{i}^{*}$ and $x_{j}^{*}$ is zero, $w_{i}^{*}=0$ and $x_{j}^{*}=0$ hold for all $i \in I$ and $j \in J$. This contradicts a constraint

$$
\sum_{j=1}^{m} x_{j}=1
$$

and hence, the solution to (1) satisfies $w^{*}>0$ and $x^{*}>0$.

Next we prove the uniqueness of the solution. Since the objective function of (1) is convex quadratic, if there is two different solutions $\left(w^{*}, x^{*}\right)$ and $(\bar{w}, \bar{x})$, all points between these two solutions also a solution to (1). Furthermore, the value of the objective function is invariable on the line passing through these two solutions.

This can be shown as follows. If we denote $z=\left(w^{T}, x^{T}\right)^{T}$, the objective function of (1) is rewritten as $f(z)=\frac{1}{2} z^{T} M z$, where $M$ is an appropriate $(n+m) \times(n+m)$ matrix. Since

$$
f\left(z^{*}\right)=f(\bar{z})=f\left(\lambda \bar{z}+(1-\lambda) z^{*}\right)
$$

holds for $0 \leq \lambda \leq 1$, we must have

$$
\begin{aligned}
\frac{1}{2}\left(z^{*}\right)^{T} M z^{*} & =\frac{1}{2}\left(\lambda \bar{z}+(1-\lambda) z^{*}\right)^{T} M\left(\lambda \bar{z}+(1-\lambda) z^{*}\right) \\
& =\frac{1}{2}\left(z^{*}\right)^{T} M z^{*}+\lambda\left(z^{*}\right)^{T} M\left(\bar{z}-z^{*}\right)+\frac{1}{2} \lambda^{2}\left(\bar{z}-z^{*}\right)^{T} M\left(\bar{z}-z^{*}\right) .
\end{aligned}
$$

However, in order to hold (4) for $0 \leq \lambda \leq 1$, we must have

$$
\left(z^{*}\right)^{T} M\left(\bar{z}-z^{*}\right)=\left(\bar{z}-z^{*}\right)^{T} M\left(\bar{z}-z^{*}\right)=0
$$

and hence, it follows from (4) that $f\left(z^{*}\right)=f\left(\lambda \bar{z}+(1-\lambda) z^{*}\right)$ holds for all $\lambda$.

Therefore, all points included in a feasible set with on the line passing through $\left(w^{*}, x^{*}\right)$ and $(\bar{w}, \bar{x})$ are solutions to (1). However, at least one of either $w_{i}^{*}$ or $x_{j}^{*}$ is zero on the boundary of the line. This contradicts the fact $w^{*}>0$ and $x^{*}>0$ shown in the first part of the proof. Thus the solution to (1) is unique.

Q.E.D.

When relative estimate of alternatives among all decision-makers is equivalent, the relative estimate of group must to equal to that of every decision-makers. The next theorem guarantees this fact.

Theorem 2. If local weights of individual decision-makers satisfy $a_{i j}^{k} / a_{i j^{\prime}}^{k}=a_{i^{\prime} j}^{k} / a_{i^{\prime} j^{\prime}}^{k}$ for all nonzero $a_{i j}^{k}, a_{i^{\prime} j}^{k}, a_{i j^{\prime}}^{k}$ and $a_{i^{\prime} j^{\prime}}^{k}\left(i, i^{\prime} \in I, j, j^{\prime} \in j\right)$, then the group weights $x^{k}$ obtained by the problem (1) also satisfies $x_{j}^{k} / x_{j^{\prime}}^{k}=a_{i j}^{k} / a_{i j^{\prime}}^{k}$ for all $i \in I$ and $j, j^{\prime} \in J$.

Proof. Under the assumption of the theorem, it is easy to see that there exist nonzero $w_{i}^{*}$ and $x_{j}^{*}$ such that $a_{i j}^{k} w_{i}^{*}=x_{j}^{*}$ holds for all $a_{i j}^{k}>0$. For such $w_{i}^{*}$ and $x_{j}^{*}$, the objective function of (1) equals to zero, and hence, $w_{i}^{*}$ and $x_{j}^{*}$ are optimal solution to (1). On the other hand, from Theorem $1, w_{i}^{*}$ and 
$x_{j}^{*} \quad$ is the unique solution to

(1).

Q.E.D.

\subsection{Aggregation of weights of criteria}

In ANP, local weights of criteria are to depend on each alternative. It is natural to consider that, in group decision-making, local weights of decision-makers for criteria are dependent to the set of alternatives evaluated by him/her. Hence, in the proposed method, first group pairwise comparison matrices of criteria for evaluating an alternative $A_{j}$ are made by geometrical averages of comparison matrices of only decision-makers who evaluate $A_{j}$. Then from these matrices, the local weights of criteria are calculated by the maximal eigenvector method.

We again consider a group decision-making problem whose evaluation graph is shown in Figure 1 . In the example, an alternative $A_{1}$ is evaluated by $D M_{1}$ and $D M_{2}$ only. So the pairwise comparison matrix for estimating $A_{1}$ is made by taking geometrical average of the pairwise comparison matrices of $D M_{1}$ and $D M_{2}$ only. Also the pairwise comparison matrix for estimating $A_{3}$ is made from the comparison matrices of $D M_{2}$ and $D M_{3}$. On the other hand, since all decision-makers evaluate the alternative $A_{2}$, the comparison matrix for $A_{2}$ as a group is made from comparison matrices of all decision-makers. In this case, the proposed method is equivalent to usual geometrical average method.

\subsection{Evaluation of global weights}

By using the aggregation method of criteria proposed in the previous subsection, local weights of criteria may be different in each alternative. In this case, the additive sum usually used in AHP is not available to evaluate the global weights. So we use the supermatrix method of ANP for evaluating global weights, because the supermatrix method permits to use different weights of criteria for each alternative.

If we denote $x_{j}^{k}$ as aggregated local weights of $A_{j}$ under a criterion $C_{k}$ and $c_{k}^{j}$ as aggregated local weights of $C_{k}$ for each alternative $A_{j}$, then the supermatrix $S$ is written by

$$
S=\left(\begin{array}{cccccc}
0 & \cdots & 0 & c_{1}^{1} & \cdots & c_{1}^{m} \\
\vdots & \ddots & \vdots & \vdots & \ddots & \vdots \\
0 & \cdots & 0 & c_{l}^{1} & \cdots & c_{l}^{m} \\
x_{1}^{1} & \cdots & x_{1}^{l} & 0 & \cdots & 0 \\
\vdots & \ddots & \vdots & \vdots & \ddots & \vdots \\
x_{m}^{1} & \cdots & x_{m}^{l} & 0 & \cdots & 0
\end{array}\right) .
$$

In ANP, local weights of criteria are usually determined in every alternative by pairwise comparison from the point of view of each alternative. But if local weights of criteria for each alternative are already known, these local weights are permitted to use without pairwise comparison. In the proposed method, local weights of criteria for an alternative are determined from the pairwise comparison matrices of decision-makers who evaluate the alternative. So far as these weights are considered as already known weights, an aggregation by the supermatrix method seems to be reasonable. 


\section{Numerical example}

In this section, we present the results of the proposed method applied for a small example with incomplete information. Let consider the example such that four decision-makers $\left(D M_{i},(i=1, \ldots, 4)\right)$ evaluate six alternatives $\left(A_{j},(j=1, \ldots, 6)\right)$ under two criteria $\left(C_{k},(k=1,2)\right)$. In Table 1 , we show local weights of decision-makers for alternatives. In the example, $D M_{1}$ evaluates alternatives $1,2,3,4$ and 6, $D M_{2}$ evaluates 2, 5 and $6, D M_{3}$ evaluates 1 only and $D M_{4}$ evaluates alternatives 2, 3, 4 and 5. The evaluation graph of this example is shown in Figure 2 and hence, it is connected. Hence, from Theorem 1, group local weights of alternative are uniquely determined. We note that, since each decision-maker uses different normalization procedure, the sum of local weights of decision-maker 2, 3 and 4 do not equal to one.

Table 1. Local weights under criteria

\begin{tabular}{|l|llll|}
\hline$C_{1}$ & $D M_{1}$ & $D M_{2}$ & $D M_{3}$ & $D M_{4}$ \\
\hline$A_{1}$ & 0.300 & & 0.400 & \\
$A_{2}$ & 0.100 & 0.500 & & 0.500 \\
$A_{3}$ & 0.100 & & & 0.500 \\
$A_{4}$ & 0.200 & & & 1.000 \\
$A_{5}$ & & 0.500 & & 0.500 \\
$A_{6}$ & 0.200 & 0.150 & & \\
\hline
\end{tabular}

\begin{tabular}{|l|llll|}
\hline$C_{2}$ & $D M_{1}$ & $D M_{2}$ & $D M_{3}$ & $D M_{4}$ \\
\hline$A_{1}$ & 0.069 & & 0.200 & \\
$A_{2}$ & 0.414 & 0.500 & & 1.000 \\
$A_{3}$ & 0.103 & & & 1.000 \\
$A_{4}$ & 0.207 & & & 0.500 \\
$A_{5}$ & & 1.000 & & 0.500 \\
$A_{6}$ & 0.207 & 0.500 & & \\
\hline
\end{tabular}

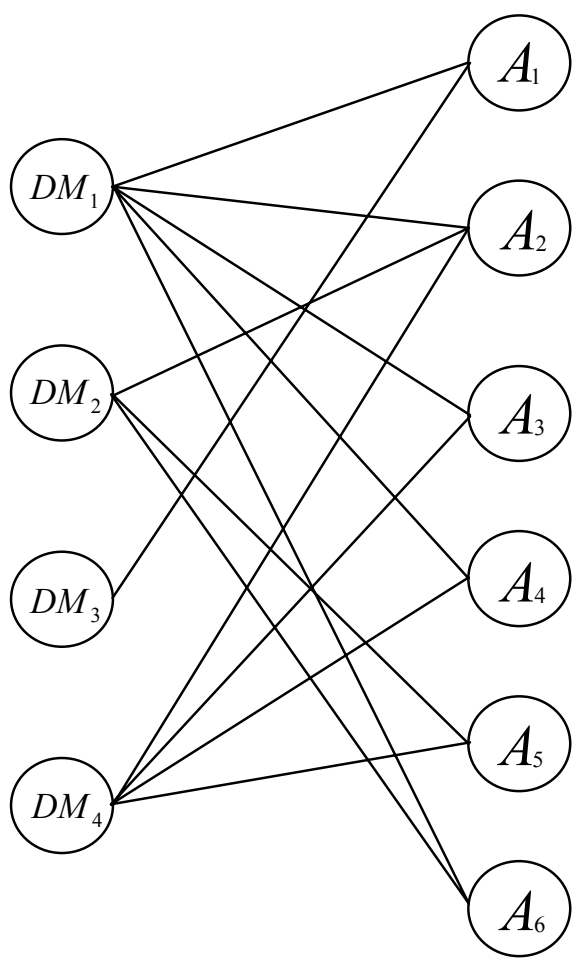

Figure 2. Evaluation graph of the example 
Table 2 shows local weights under each criterion aggregated by the optimization problem (1). It can be seen from Table 1 that local weights under $C_{1}$ are in ratio of 3:1:1:2:2:2 for all decision-makers if all blanks are filled with appropriate number. From Table 2, the aggregated local weights under $C_{1}$ are also in the same ratio, and it is verified that Theorem 2 is satisfied. When $D$ denotes the optimal value of (1), which can be considered as the dissatisfaction value of a group, $D=0$ under $C_{1}$. This means that preferences of all decision-makers are same and that there is nothing dissatisfied in a group. On the other hand, under $C_{2}$, the value of $D$ does not equal to zero because preferences of decision-makers are slightly different.

Table 2. Aggregated local weights

\begin{tabular}{|l|cccccc|cccc|l|}
\hline & $x_{1}$ & $x_{2}$ & $x_{3}$ & $x_{4}$ & $x_{5}$ & $x_{6}$ & $w_{1}$ & $w_{2}$ & $w_{3}$ & $w_{4}$ & \multicolumn{1}{c}{$D$} \\
\hline$C_{1}$ & 0.273 & 0.091 & 0.091 & 0.182 & 0.091 & 0.182 & 0.909 & 0.182 & 0.682 & 0.182 & 0 \\
$C_{2}$ & 0.087 & 0.227 & 0.170 & 0.147 & 0.213 & 0.156 & 0.661 & 0.270 & 0.433 & 0.231 & 0.021 \\
\hline
\end{tabular}

Next we present pairwise comparison matrices for criteria of each decision-maker in Table 3. In this example, since $A_{1}$ is evaluated by $D M_{1}$ and $D M_{3}$, local weights of criteria for evaluating $A_{1}$ in a group are determined from comparison matrices of $D M_{1}$ and $D M_{3}$, which is shown in Table 4. Local weights of criteria for each alternative can be determined in a similar way and the results are collected in Table 5 .

Table 3. Pairwise comparison matrices of criteria

\begin{tabular}{|c|cc|}
\hline$D M_{1}$ & $C_{1}$ & $C_{2}$ \\
\hline$C_{1}$ & 1 & 1 \\
$C_{2}$ & & 1 \\
\hline
\end{tabular}

\begin{tabular}{|c|cc|}
\hline$D M_{2}$ & $C_{1}$ & $C_{2}$ \\
\hline$C_{1}$ & 1 & 3 \\
$C_{2}$ & & 1 \\
\hline
\end{tabular}

\begin{tabular}{|c|cc|}
\hline$D M_{3}$ & $C_{1}$ & $C_{2}$ \\
\hline$C_{1}$ & 1 & 2 \\
$C_{2}$ & & 1 \\
\hline
\end{tabular}

\begin{tabular}{|c|cc|}
\hline $\mathrm{DM}_{4}$ & $C_{1}$ & $C_{2}$ \\
\hline$C_{1}$ & 1 & $1 / 2$ \\
$C_{2}$ & & 1 \\
\hline
\end{tabular}

Table 4. Weights of criteria for $A_{1}$

\begin{tabular}{|c|cc|c|}
\hline$A_{1}$ & $C_{1}$ & $C_{2}$ & Weight of criterion \\
\hline$C_{1}$ & 1 & $\sqrt{1 \times 2}$ & 0.586 \\
$C_{2}$ & & 1 & 0.414 \\
\hline
\end{tabular}

Table 5. Weights of criteria for each alternative

\begin{tabular}{|c|c|c|c|c|c|c|}
\hline & $A_{1}$ & $A_{2}$ & $A_{3}$ & $A_{4}$ & $A_{5}$ & $A_{6}$ \\
\hline$C_{1}$ & 0.586 & 0.551 & 0.414 & 0.414 & 0.551 & 0.634 \\
\hline$C_{2}$ & 0.414 & 0.449 & 0.586 & 0.586 & 0.449 & 0.367 \\
\hline
\end{tabular}

From the results of Tables 2 and 5, we have constructed a supermatrix and have calculated global weights. The results are shown in Table 6 . In the calculation, we have used the power method written in (Taji et al. 1999). It is observed from Table 6 that the weight of $A_{6}$ is largest and the group thinks of $A_{6}$ most important among alternatives. 


\section{Conclusion and future researches}

In this paper, we have proposed a new group decision-making model, which is available to problems with incomplete information. In the proposed method, local weights of alternatives are aggregated as a solution to a quadratic programming problem (1). Local weights of criteria are aggregated in each alternative. Hence weights of criteria for each alternative may be different and the supermatrix method in ANP is used to evaluate global weights. A small numerical example has shown that the proposed method can calculate global weights of the problem with incomplete information.

The proposed method is considered as an extension of ANP to group decision-making problems. But this paper is only a starting point of this approach and we need further study for applying to practical decision-making problems. Some subjects of future research are following.

- In the proposed method, the problem (1) is used to aggregate local weights of alternatives. Some other methods such as stress methods (Nakanishi and Kinoshita, 1999) and large-scale AHP (Yamaki and Sekitani, 1999) are also available, and we need compare with these methods.

- We use the geometrical average method to aggregate local weights of criteria. It is also needed to apply some other aggregation method to this part.

- In the proposed method, there are only two hierarchies, alternatives and criteria. The method only takes a so-called outer dependence case into account. But it is often seen in decisionmaking problems that criteria have multiple hierarchical structure and that there is dependent relation between two or more criteria. We need an extension of this model available to treat these cases.

\section{References}

Harker, P. T. (1987), "Incomplete pairwise comparisons in the Analytic Hierarchy Process," Mathematical Modelling, 9, 837-848.

Harker, P.T. and Vargas, L.G. (1987), "The Theory of Ratio Scale Estimation: Saaty's Analytic Hierarchy Process," Management Science, 33, 1383-1403.

Nakanishi, M. and Kinoshita, E. (1999), "Inter-Viewpoint Stress Methods and Group Decision Making Stress Method," Proceedings of the Fifth International Symposium on the Analytic Hierarchy Process, Kobe International Conference Center, Kobe, Japan, August 12-14, 290.

Saaty, T. L. (1980), The Analytic Hierarchy Process, McGraw Hill, New York.

Saaty, T. L. (1996), The Analytic Network Process, RWS Publications, Pittsburgh, PA.

Taji, K., Sagayama, Y. and Tamura, H. (1999), "On a Revised Analytic Network Process (ANP) for Avoiding Irrational Rank Reversal - A Revised Normalization Procedure and Calculation Method -," Proceedings of the Fifth International Symposium on the Analytic Hierarchy Process, Kobe International Conference Center, Kobe, Japan, August 12-14, 141-146.

Yamada, Y., Sugiyama, M. and Yamaki, N. (1997), “Group Analytic Hierarchy Process Based on Consensus Making Model," (in Japanese) Journal of Operations Research Society of Japan, 40, 236-244.

Yamaki, N. and Sekitani, K. (1999), "A Large-scale AHP Including Incomplete Information and Multiple Evaluators and Its Application to Personnel Administration Evaluation System," (in Japanese) Journal of Operations Research Society of Japan, 42, 405-421. 
